\title{
Iatrogenic Damage to the Periodontium Caused by Removable Prostho- dontic Treatment Procedures: An Overview
}

\author{
Khaja Amjad Hussain ${ }^{1, *}$, Saleh Nasser Azzeghaibi ${ }^{2}$, Bassel Tarakji ${ }^{1}$, SenthilRajan R. S. ${ }^{3}$, \\ Syed Sirajuddin ${ }^{3}$ and Sandeep S. Prabhu ${ }^{3}$
}

\author{
${ }^{I}$ Department of Oral-maxillofacial Science, Alfarabi College of Dentistry and Nursing, Riyadh, Saudi Arabia; ${ }^{2}$ Director \\ of Alfarabi College of Dentistry and Nursing, Riyadh, Saudi Arabia; ${ }^{3}$ Department of Periodontology, Rajarajeswari \\ Dental College \& Hospital, Bangalore-560074. Karnataka, India
}

\begin{abstract}
As the number of aged people in the world is growing, the need to provide patients with tooth alternate through removable partial dentures is equally growing. There are adversarial effects that should be kept in mind which might disturb the remaining teeth; specially, the abutments and the supporting tissues. These effects might spread to the muscles of mastication and also to the supporting periodontal tissues. Thus, we should plan removable partial dentures (RPDs) without mutilation to the adjacent teeth or the underlying tissues.
\end{abstract}

Keywords: Iatrogenic damage, periodontium, removable prosthodontic treatment.

\section{INTRODUCTION}

While it is established that meticulous oral hygiene instructions play a key role in decreasing the risks linked with removable partial dentures, the inherent capability of removable partial dentures to hold dental plaque is still considered a chief risk factor and might explain the latest link between the recommendation of partial dentures and the progress of periodontitis around the abutment teeth $[1,2]$. This should encourage dental researchers to develop material with low abilities for plaque adhesion.

The other adverse effects of a partial denture as a result of plaque buildup is the progress of severe inflammation (Fig. 1) of the underlying tissues developing to denture stomatitis, or as an outcome of direct trauma to the gums and other soft tissues might lead to bleeding and bad mouth odor [3].

This type of inflammation (Fig. 2) is still related to the capacity of patients to follow oral hygiene instructions and retain their denture clean and hygienic.

RPDs can upturn the occurrence of caries, damage the periodontium, and increase the amount of trauma on the natural teeth [4]. These changes are ascribed to poor oral hygiene, increased plaque, calculus buildup and transmission of excessive forces to the periodontal structures from occlusal surfaces of the framework of RPDs [5].

Many partial denture framework designs add to improved alteration in the oral microflora and dental plaque accumulation [6]. Mihalow and Tinanoff after prosthesis placement observed an increased number of Streptococcus mutans in the saliva of RPD patients [7]. This finding recommends that

\footnotetext{
*Address correspondence to this author at the Alfarabi college of Dentistry and Nursing, Riyadh, Saudi Arabia; Tel: 00966227 3151; Fax: 00966232 4580; E-mail: amjadh@gmail.com
}

for patients receiving RPDs chemoprophylactic approaches need to be established to control and lessen biofilm formation and caries development, and thus helping to maintain the patient's oral health [8].

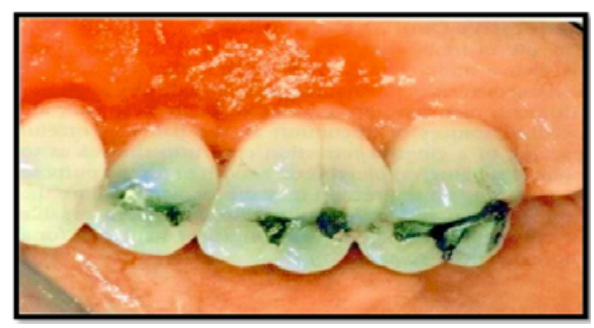

Fig. (1). Inflamed palatal gingiva associated with a maxillary provisional acrylic partial denture.

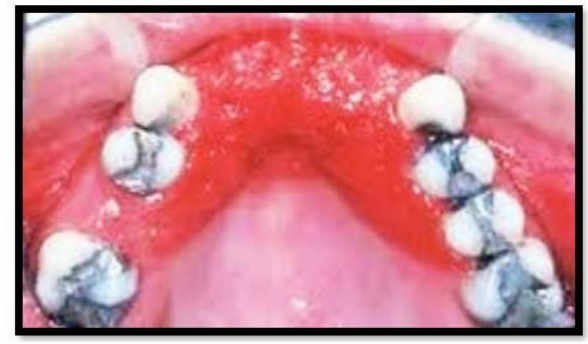

Fig. (2). Plaque accumulation underneath partial denture leading to development of severe inflammation of the underlying tissues.

The relation between the opposing effects of partial dentures on abutment teeth (Fig. 3) is generally correlated to the type of the denture material used whether it has an acrylic or cobalt- chromium (Co-Cr) base. This is essential as the ability of a denture to hold plaque is related to the type of denture base; a Co-Cr denture is more hygienic. However, the capacity of patient to keep the oral hygiene ideal after den- 
ture addition is the main reason which affects the success of removable denture whether it is acrylic or Co-Cr. Accumulation of plaque about and beneath the various components of a partial denture is not only accountable for developing chronic periodontitis but can effect in gingival recession and root caries [9].

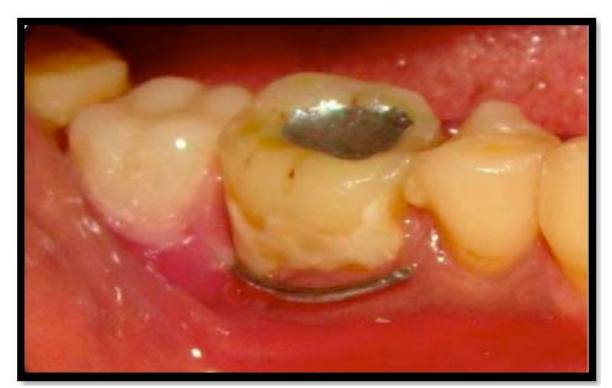

Fig. (3). Removable partial denture in relation to 47 showing clasp on gingival margin and rough class $\mathrm{V}$ filling.

Another constituent of $\mathrm{Co}-\mathrm{Cr}$ denture is the major connector which allows distributing the occlusal load to the neighboring teeth. Using sectional or poorly planned partial dentures has led to undesirable damage to neighboring teeth and also might lead to the unexpected accident of denture swallowing. It is the dentist's obligation to be aware of the possible damage to the left over teeth, especially abutments and any harm that might be happening from unexpected inhaling these types of undersized removable partial dentures.

Aspiration and inadvertent swallowing of dental prostheses has been reported frequently in the literature. Aspirated or swallowed dental prosthesis can be accomplished radiographically unless the prostheses is made entirely of acrylic resins, where it is difficult. In adults aspiration of dental restorations and teeth is recognized, although it is an uncommon finding in the literature. The reasons for for aspiration could be attributed to maxillofacial trauma, dental treatment procedures or ethanol intoxication and dementia. Therefore, removable partial dentures should be designed not only to preserve the remaining teeth but also to prevent accidental swallowing of these appliances.

\section{EFFECTS OF RPDS ON PERIODONTAL INDICES}

\section{Effects on Plaque}

Several studies have shown the effect of RPDs on the quantity and quality of plaque. A study done by ChamrawyE showed greater plaque formation on teeth that are in contact with RPDs and iterated the need for patient education in keeping the endangered teeth clean.

A study done by ChamrawyE showed that the RPDs promoted the proliferation of spirochetes and spirilla over short rods and cocci, thereby altering the composition of plaque. They concluded that by intense toothbrushing the level of plaque can be kept low. Brushing after each meal, use of special toothbrushes for proximal surfaces, and frequent cleaning of the dentures are the hygiene measures to be followed. Forty six RPDs and their effect on plaque accumulation was studied by one group of researches and they concluded that the design of the denture design should simple covering only the essential hard and soft tissues and a higher level of oral hygiene is needed for RPD patients.
Comparable observations were made in a 1-year study of 3 maxillary RPD designs. The study concluded that both clinically and histologically, the gingival areas that are covered by parts of the RPD without relief showed the most unfavorable periodontal reactions; whereas, the areas that were uncovered were least affected. Based on the results, the proposed distance for all RPD components was 5 to $6 \mathrm{~mm}$ away from the gingival margins. Cingulum bar showed fewer detrimental effects on gingival tissues than the lingual apron major connector as noticed in a short-term, single-blind cross-over experimental gingivitis trial. The more plaque accumulation was due to the increased tissue coverage by the latter major connector. Another group of investigators suggested simpler RPD designs with less tissue coverage and frequent recalls are needed as extra hygiene measures as the ecologic changes brought about by RPDs are not offset by toothbrushing as it was commonly practiced. A number of clinical studies have concluded strict recall and oral hygiene resulted in proper plaque control in RPD wearers.

\section{EFFECTS ON FORCES EXERTED ON TEETH AND TOOTH MOBILITY}

Biomechanical aspects of RPD design has always been a concern in the literature review. Unilateral or bilateral distal extension RPDs shares their support between the edentulous ridge and abutment teeth. It has been reported that RPD design affects the distribution of force on residual alveolar ridges and abutment teeth.

Reduction of stress on the abutment teeth is dependent of the rigidity of major connectors and maximum coverage of denture-bearing areas with denture bases. This study also showed that as the periodontal support diminishes due to increase in stress concentration on abutment teeth. One group of investigators used intraoral strain gauges to measure the lateral horizontal forces applied to abutment teeth by RPDs during function and noted that the forces exerted on abutment teeth during swallowing were almost twice those exerted during mastication on a daily basis.

Another investigator stressed apart from the occlusal forces, the tongue, cheeks, and lips contribute to generating torque and forces exerted on abutment teeth. The literature suggests intracoronal attachment designs produced more torque on abutment compared to clasp-retained designs. Clinical studies suggested that as the denture-wearing period proceeds, there was reduction of torque exerted on abutment teeth. This "settling" period lasts about 1 to 1.5 months from the time of insertion of new RPDs. This reduction in the torque on the abutment can be attributed to changes of jaw movement in the frontal plane, properties of the alveolar mucosa, adaptation of the oral tissues to the denture, or changes in the chewing points of the RPDs.

Mobility of abutment teeth measured intraorally in a 200day experiment using two different RPD designs in a crossover experiment, where both RPDs were mandibular distal extension, anchored on the canines was carried out. The first design consisted of a buccal cast circumferential clasp arm and mesioocclusal rest, while the second design consistedan elastic wire clasp arm with no rest seat. Both designs elicited significant acute or gradual changes of abutment tooth mobility. There was no mention of control measures or maintenance, and the small sample size precluded any definite con- 
clusions regarding design influence on the changes observed. Other investigators have reported properly designed RPDs over a 2-year period helped in stabilizing periodontally mobile teeth. They also reported rigid major connectors along with parallel guide planes are a prerequisite for success of an RPD. Complete palatal coverage was used on the maxillary arch, and "fingers" were placed over the incisal edges of the mandibular anterior teeth. However, this report produced no control or proper statistics.

A 4-year longitudinal study of RPD patients reported that denture wearers had on average $18 \%$ of their teeth mobile and $25 \%$ with a tendency for increased mobility by the end of the study period. However, the patients who did not wear their dentures had no significant changes in tooth mobility. The study concluded that in patients with a high standard of oral hygiene, RPDs can be used for rehabilitation for longer periods without any major damage to the remaining teeth. Several investigators have reported that the forces exerted on abutment teeth are also influenced by the inclination of the residual ridge in vitro and in vivo studies. A cross-sectional study concluded that RPDs in an elderly population might be associated with increased tooth mobility, although the nature of the study precluded any definite conclusions. Several long-term clinical studies have shown that properly designed RPDs with strict oral hygiene and frequent recalls did not have any detrimental effects on tooth mobility.

\section{SPLINTED VERSUS NONSPLINTED ABUTMENTS}

Carlsson et al. suggested that the forces of the RPDs can be better withstood by splinting primary abutment teeth. Based on the observation during their 4-year clinical study, they noticed no deterioration of the periodontal condition on the splinted abutments.

An in vitro study of a photo elastic model demonstrated that fixed splinting of adjacent abutment teeth is an important factor when attachment retainers are used for an extension RPD. Similar results were obtained in a study which demonstrated strains induced on abutment teeth when extracoronal attachments are used in distal extension RPDs. Strain guage was used in anvitro model which suggested that at least two teeth should be splinted for reduction of stresses. Another study with regards to same issue concluded a distal abutment with moderate periodontal support should be splinted to one sound adjacent tooth to decrease the load transfer by a distal extension RPD. So far, no clinical studies have compared splinted and non-splinted abutments for RPDs.

No clinical evidence is available to indicate that torquing forces transmitted to the abutment teeth from distal extension RPDs posed any threat to their periodontal status, provided that oral hygiene is maintained. Laboratory models cannot accurately predict actual forces in vivo and their effects on oral structures. Literature has shown that traumatic forces on teeth do not cause attachment loss in the absence of plaque and inflammation. An initial increase in tooth mobility may be the result of adaptive, non-pathologic changes. However, fabrication of RPDs should follow sound principles to minimize stresses. The framework should be adjusted properly, and distal extension bases should be constructed using an altered cast.

\section{CONCLUSION}

The detrimental changes in the quality and quantity of plaque due to the use of RPDs can be reduced by implementation of scrupulous hygiene of both oral cavity and dentures. Factors that affect force distribution from the RPD to the abutment teeth and edentulous ridge include denture design, residual ridge inclination, and denture base adaptation. Properly designed and maintained RPDs can endow with longterm clinical service without any detrimental effects on the periodontal condition of the remaining dentition, by establishing pre-prosthetic periodontal health and meticulous oral hygiene. A good long-term prognosis can be established by frequent hygiene recalls and prosthetic maintenance.

\section{CONFLICT OF INTEREST}

The authors confirm that this article content has no conflict of interest.

\section{ACKNOWLEDGEMENTS}

Declared none.

\section{REFERENCES}

[1] Behr M, Zeman F, Passauer T, Koller M, Hahnel S. Clinical performance of cast clasp-retained removable partial dentures: a retrospective study. Int J Prosthodont 2012; 25: 138-44.

[2] Emami E, Taraf H, de Grandmont P, Gauthier G, de Koninck L. The association of denture stomatitis and partial removable dental prostheses: a systematic review. Int J Prosthodont 2012; 25: 113-9.

[3] Amaral BA, Barreto AO, Seabra EG, Roncalli AG, da Fonte Porto Carreiro A. A clinical follow-up study of the periodontal conditions of RPD abutment and non-abutment teeth. J Oral Rehabil 2010; 37 : 545-52.

[4] Carlsson GE, Hedegard B, Koivumaa KK. Studies in partial dental prosthesis, III. A longitudinal study of mandibular partial dentures with double extension saddles. Acta Odontol Scand 1962; 20: 95119.

[5] Rocha EP, Francisco SB, Del BelCury AA, Cury JA. Longitudinal study of the influence of removable partial denture and chemical control on the levels of Streptococcus Mutans in saliva. J Oral Rehabil 2003; 30: 131-8.

[6] Mihalow DM, Tinanoff N. The influence of removable partial dentures on the level of Strepto-coccus Mutans in saliva. J Prosthet Dent 1988; 59: 49-51.

[7] Amaral BA, Barreto AO, Gomes Seabra E, et al. A clinical followup study of the periodontal conditions of RPD abutment and nonabutment teeth. J Oral Rehabil 2010; 37: 545-52.

[8] Rocha EP, Francisco SB, Del Bel Cury AA, Cury JA. Longitudinal study of the influence of removable partial denture and chemical control on the levels of Streptococcus Mutans in saliva. J Oral Rehabil 2003 ; 30:131-8.

[9] Cooke LD, Baxter PW. Accidental impaction of partial dental Prostheses in the upper gastrointestinal tract. Br Dent J 1992; 172: 451-2.

(C) Hussain et al.; Licensee Bentham Open.

This is an open access article licensed under the terms of the Creative Commons Attribution Non-Commercial License (http://creativecommons.org/licenses/by-nc/3.0/) which permits unrestricted, non-commercial use, distribution and reproduction in any medium, provided the work is properly cited. 